\title{
THE CHOICE OF REVERSE LOGISTICS RECYCLING MODEL IN THE NONLINEAR DEMAND
}

\section{LEPING ZHOU and SHENGJIA XUE}

Education College of Jinan University

P. R. China

e-mail:13112443@qq.com

tlowzy@jnu.edu.cn

\begin{abstract}
We discuss the choice of recycling mode of reverse logistics under nonlinear demand. In determining bilateral monopoly market and leading manufacturer of the closed-loop supply chain recycling mode were analyzed, based on the game theory of Stackelberg equilibrium, constructing reverse logistics three basic recovery mode -- the manufacturer for recycling, reclaiming retailers, and third-party recovery of nonlinear programming model, through mathematical derivation were decision analysis and lingo software simulation verification. It is pointed out that the retailer is responsible for recycling is the best way to recovery, and finally the optimal model of optimization.
\end{abstract}

\section{Introduction}

With the exhaustion of the sources of the earth and the people environmental protection consciousness enhancement, the waste products of recycling has become a kind of new logistics mode-reverse logistics mode. In the reverse logistics of three types (recycling, re-use, and re-manufacturing), based on re-manufacturing reverse logistics has greater complexity and higher economic value, where in the collector 2010 Mathematics Subject Classification: 90b06.

Keywords and phrases: nonlinear demand, reverse logistics, recycling mode, Stackelberg equilibrium, nonlinear programming.

Received April 20, 2016

(C) 2016 Scientific Advances Publishers 
with the mode of selection is a research hotspot. At present, the research of it has made abundant achievements [1-8], the most representative is Savaskan etc. [1, 2] of work, they are considered three kind of recyclings patterns, namely, the manufacturer direct recovery, reclaiming the retailer entrusted by the manufacturer, the manufacturer shall entrust a third party company for recovery, use game-theoretic models to study how the members of the enterprise in different recovery mode of the optimal decision-making. However, the research is all about the market price of the linear function is done under the assumption of. In real life, of one-of-a-kind product needs more representation is that the price of the non-linear function. This paper is to put forward the demand for recycling mode of decision-making.

\section{Non-linear Demand Function}

Demand function in the representation of a certain market size, in the actual commodity markets, each product will have a certain amount of market capacity. In the form of linear demand function, such as Savsakan. As shown in Figure 1.

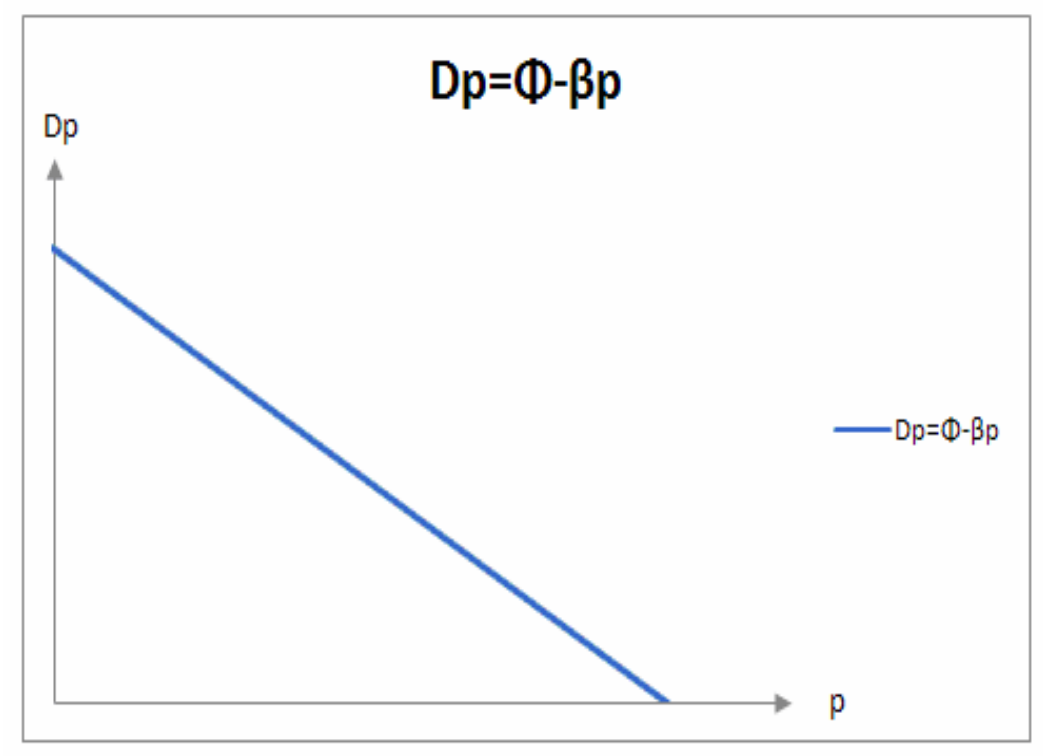

Figure 1. Function diagram: $D(p)=\phi-\beta p$. 
When $\alpha<1$, as shown in Figure 2, when the price is relatively low, the demand for commodities as prices change rapidly, when the commodity price is too high, the demand for commodities subject to price influence is not obvious. In a certain category of pricing represents change rate of demand price elasticity of demand is less than the lack of goods, such as some necessities of life.

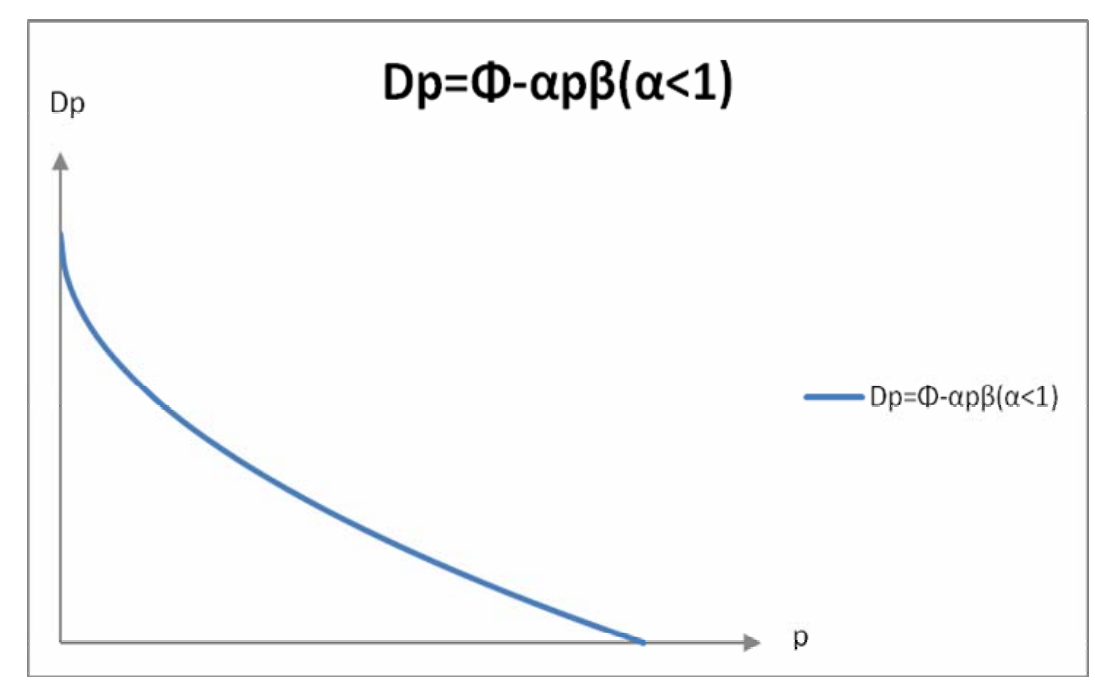

Figure 2. Function diagram: $D(p)=\phi-\beta p^{\alpha}(\alpha<1)$.

When $\alpha>1$, as shown in Figure 3, when the price is relatively low, the demand for goods by the price is not obvious, in a certain range of prices, with the increase in prices, the demand for goods decreased rapidly. The proportion of the change in demand is greater than the proportion of the price change, which represents the commodity is flexible. Such as electronic products, mobile phones, television, and other household electrical appliances, is the main type of reverse logistics research. 


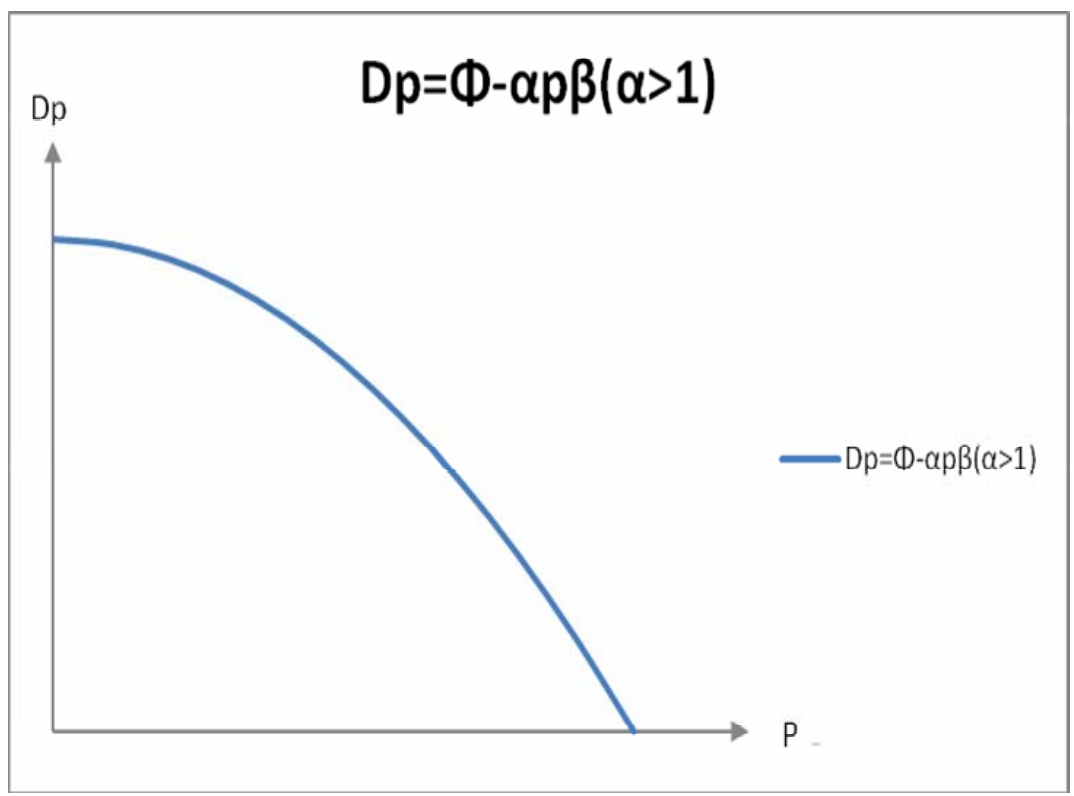

Figure 3. Function diagram: $D(p)=\phi-\beta p^{\alpha}(\alpha>1)$.

In view of the complexity of nonlinear computation, we make $\alpha=2$ the research in this paper to determine the market demand function $D(p)=\phi-\beta p^{2}$ in order to carry out relevant research and analysis work.

\section{Problem Description and Basic Assumptions}

In this paper, the basic meanings of the symbols: $C_{m}$ is raw materials for manufacturing a new product cost, $C_{r}$ is the cost of new products for remanufacture of waste products into and new products; $C_{r}<C_{m} ; p$ is sales price; $\varpi$ is the manufacturer's wholesale price provide to retailers; $A$ is the recycling price of waste products, $b$ is the pay price of for manufacturers to recycle waste products, obviously $A \leqslant b \leqslant \Delta$, where $\Delta=C_{m}-C_{r} ; \tau$ is recovery rate, $0 \leq \tau \leq 1$; according to [1, 6] $I=L \tau^{2}, L>0, I$ is the fixed investment of recycling, $L$ is the 
scale parameter, obviously the average cost of the manufacturer is $C_{m}(1-\tau)+C_{r} \tau=C_{m}-\Delta \tau ; A$ the average cost of the recycling waste products, including the recycle price paid to consumer and the average cost of transportation in order to ensure the manufacturers can make profit from recycling and re-manufacturing; here we assume $O \leq A \leq \Delta, A$ is exogeneous, does not affect the value of demand.

$\prod_{i}^{M, j}$ is the profit function of $i$ under $j$ recycle model, where $i=m, R, 3 P, t$, respectively, which indicated that manufacturers, retailers, and the third party and the overall profits; $j=M, R, 3 P$. All variables and functions of superscript $*$ said optimal solution and optimal value.

Assumptions are as follows: product demand function $D(p)=\phi-\beta p^{2}$; market is leading manufacturer (in fact it is usually true). Thus, the manufacturer can as a leader in Stackelberg game; all parameters and variables are greater than zero, meet and the sales price $C_{m} \leq p \leq \bar{P}, \bar{P}$ which price bound (the actual calculation desirable for larger positive).

Can be seen from the basic symbols and assumptions: the number of recyclable products can be recycled $\tau D(p)=\tau \alpha p^{-\beta}$, the total cost of recycling is $L \tau^{2}+A \tau D(p)=L \tau^{2}+A \tau \alpha p^{-\beta}$.

\section{Study on Three Models of Recovery}

Under the bilateral monopoly market, the reverse logistics of the closed loop supply chain can be divided into direct recycling of manufacturers, manufacturers commissioned by the manufacturers to recycle, manufacturers commissioned third party recovery. Related models are constructed as follows: 


\subsection{Manufacturers direct recovery- $(M, M)$ nonlinear programming problems}

In the model, the manufacturer is responsible for the production of new products, recycling of waste products and the production of new products, retailers are only responsible for sales. Stark and Berg game decision making process: the manufacturer is the leader in Stackelberg, retailers as a follower, manufacturers determines a wholesale price $w$ and recovery $\tau$ according to the reaction function of retailers, retailers selling price $p$ is determined according to the manufacturer's decision. We have: for a given wholesale price $w$, the retailer's optimal response to the solution:

$$
\underset{p}{\operatorname{Max}} \prod_{R}^{M, M}=\left(\phi-\beta p^{2}\right)(p-w)
$$

such as pseudo concave analysis shows that, $\prod_{R}^{M, M}$ the price $P$ is pseudo concave, so best response equivalent to the first-order conditions:

$$
\frac{\partial \prod_{R}^{M, M}}{\partial p}=0,
$$

manufacturers according to the retailer's reaction function determine the wholesale price and return rate, the profit function:

$$
\prod_{M}^{M, M}=\left(\phi-\beta p^{2}\right)\left(w-c_{m}+(\bar{\Delta}-A) \tau\right)-L \tau^{2} .
$$

Taking (4.3) as the objective function, considering (4.2) and other assumptions, the range of recovery rate is between 1 and 0 as the corresponding constraints, so as to construct the following nonlinear programming problem:

$$
\underset{p . w . \tau}{\operatorname{Max}} \prod_{M}^{M, M}=\left(\phi-\beta p^{2}\right)\left(w-c_{m}+(\bar{\Delta}-A) \tau\right)-L \tau^{2},
$$




$$
(M, M) \text { s.t. }\left\{\begin{array}{l}
3 \beta p^{2}-2 \beta p w-\phi=0, \\
w+(\bar{\Delta}-A) \tau \geq c_{m}, \\
0 \leq w \leq p \leq \bar{p}=\sqrt{\frac{\phi}{\beta}} \\
p \geq 0, \\
0 \leq \tau \leq 1 .
\end{array}\right.
$$

The manufacturer is responsible for the recovery of the total profit function under the model

$$
\prod_{T}^{M, M}=\prod_{M}^{M, M}+\prod_{R}^{M, M}
$$

Because it is a nonlinear programming, cannot find out the variables of the analytical solution, we can use the Lagrange multiplier method to establish the relationship between variables. Due to various constraints on the optimal solution of inequality is "Relaxation", using Lagrange multiplier $\lambda$, constructing a new function:

$$
\Gamma_{p, w, \tau, \lambda}=\left[\left(\phi-\beta p^{2}\right)\left(w-c_{m}+(\bar{\Delta}-A) \tau\right)-L \tau^{2}\right]+\lambda\left(3 \beta p^{2}-2 \beta w p-\phi\right),
$$

where $p, w, \tau, \lambda$ for new function variables, respectively, on these variables for partial derivative (4.6), (4.8), (4.10) and (4.12), ordered the first order partial derivatives of 0 , relation (4.7), (4.9), (4.11), (4.13) and (4.14).

$$
\frac{\partial \Gamma}{\partial p}=-2 \beta p\left(w-c_{m}+(\bar{\Delta}-A) \tau\right)+6 \lambda \beta p-2 \lambda \beta w .
$$

Order $\frac{\partial \Gamma}{\partial p}=0$, that is,

$$
\begin{gathered}
{\left[3 \lambda-\left(w-c_{m}+(\bar{\Delta}-A) \tau\right)\right] p-\lambda w=0} \\
\left.\frac{\partial \Gamma}{\partial w}=\phi-\beta p^{2}-2 \lambda \beta p\right) .
\end{gathered}
$$


Order $\frac{\partial \Gamma}{\partial w}=0$, that is,

$$
\begin{gathered}
\lambda=\frac{\phi-\beta p^{2}}{2 \beta p}, \\
\frac{\partial \Gamma}{\partial \tau}=\left(\phi-\beta p^{2}\right)(\bar{\Delta}-A)-2 L \tau .
\end{gathered}
$$

Order $\frac{\partial \Gamma}{\partial \tau}=0$, that is,

$$
\begin{gathered}
\tau=\frac{\left(\phi-\beta p^{2}\right)(\bar{\Delta}-A)}{2 L}, \\
\frac{\partial \Gamma}{\partial \lambda}=3 \beta p^{2}-2 \beta w p-\phi .
\end{gathered}
$$

Order $\frac{\partial \Gamma}{\partial \lambda}=0$, that is,

$$
\begin{gathered}
p=\frac{\beta w+\sqrt{\beta^{2} w+3 \beta \phi}}{3 \beta}, \\
w=p-\frac{\phi-\beta p^{2}}{2 \beta p} .
\end{gathered}
$$

We will get to the conversion, could also be used to calculate values of each variable to obtain, (4.9), (4.11), (4.13) and (4.14) substitution (4.7) type equation, we derive this equation:

$$
\begin{aligned}
2(\bar{\Delta}-A)^{2} \beta^{3} p^{5}-3(2+L) \beta^{2} p^{4}+ & {\left[4 \beta^{2} C_{m} L-2(\bar{\Delta}-A) \beta^{2} \phi\right] p^{3} } \\
+ & 2 \beta \phi(3-L) p^{2}+\phi^{2} L=0 .
\end{aligned}
$$

There is no formula solution for one yuan and five equations. We cannot find the analytic formula of each variable value $p$. But these relationships can help us to derive a comparison of the size of each target value in the following. 


\subsection{Retailer responsible for recycling-( $(M, R)$ nonlinear programming problem}

The retailer is responsible for recovering the model, the retailer is responsible for sales of new products and recycling of waste products, manufacturer is responsible for the manufacture of new products, manufacturers need to through the payment products recycling compensation price $b$ from the hands of the retailers get recycled products remanufacturing. The decision-making process is under Stackelberg game decision framework, manufacturers first compensation price $b$ and the wholesale price of $w$ is determined according to the reaction function of retailers, retailers according to the manufacturer's decision to determine the sales price $p$ and recovery rate of $\tau$, the retailer's reaction for solving:

$$
\underset{p, \tau}{\operatorname{Max}} \prod_{R}^{M, R}=\left(\phi-\beta p^{2}\right)(p-w+(b-A) \tau)-L \tau^{2} .
$$

As above, $\prod_{R}^{M, R}$ has a pseudo concave on the price of $p$ and $\tau$, and the optimal reaction is equivalent to the first order partial derivative of $p$ and $\tau:$

To

$$
\begin{gathered}
\frac{\partial \prod_{R}^{M, R}}{\partial P}=0, \\
\frac{\partial \prod_{R}^{M, R}}{\partial \tau}=0,
\end{gathered}
$$

the manufacturer determines the wholesale price and the recycling price according to the retailer's response function $W$, and its profit function is

$$
\prod_{M}^{M, R}=\left(\phi-\beta p^{2}\right)\left(w-c_{m}+(\bar{\Delta}-b) \tau\right)
$$


To (4.19) as the objective function, considering the type (4.17), (4.18) and other assumptions as constraints, and the range of the recovery rate in the range of 1 to 0 , the construction of $M$ model, $R$ nonlinear programming problems are as follows:

$$
\begin{aligned}
& \underset{w, b, p, \tau}{\operatorname{Max}} \prod_{M}^{M, R}=\left(\phi-\beta p^{2}\right)\left(w-c_{m}+(\bar{\Delta}-b) \tau\right), \\
& (M, R) \text { s.t. }\left\{\begin{array}{l}
3 \beta p^{2}-2 \beta(w-(b-A) \tau) p-\phi=0, \\
\left(\phi-\beta p^{2}\right)(b-A)-2 L \tau=0, \\
0 \leq w \leq p \leq \sqrt{\frac{\phi}{\beta}} \\
A \leq b \leq \bar{\Delta}, \\
p \geq w, \\
0 \leq \tau \leq 1 .
\end{array}\right.
\end{aligned}
$$

In this model, when $b^{* R}=\bar{\Delta}$, the objective function tends to the maximum.

The total profit function is responsible for recovery in retailer model:

$$
\prod_{T}^{M, R}=\prod_{M}^{M, R}+\prod_{R}^{M, R}
$$

We have 4.1 similar calculation:

$$
\begin{gathered}
p=\frac{\beta[w-(b-A) \tau]+\sqrt{\beta^{2}[w-(b-A) \tau]^{2}+3 \beta \phi}}{3 \beta}, \\
\tau=\frac{\left(\phi-\beta p^{2}\right)(b-A)}{2 L} .
\end{gathered}
$$




\subsection{Indirect recycling of third party companies- $(M, 3 P)$ nonlinear programming problem}

Under the model of the third party responsible for recycling the manufacturer, the third party is responsible for recycling the waste products and providing it to the manufacturer. The manufacturer is responsible for manufacturing and re-creating new products, and the retailer is responsible for the sales of new products. The decision-making process is under Stackelberg game decision framework, manufacturers according to third party companies and retailers reaction function to determine the wholesale price of $w$ and to third party compensation price $b$, retailers and third-party companies according to the manufacturer's decision respectively, sales price $p$ and recovery rate of $\tau$ and the retailer's optimal response to solve:

$$
\underset{p}{\operatorname{Max}} \prod_{R}^{M, 3 P}=\left(\phi-\beta p^{2}\right)(p-w)
$$

According to the analysis of the pseudo concave, $\prod_{R}^{M, 3 P}$ has a pseudo concave to the price of $p$, so the optimal reaction is equivalent to the first order condition

$$
\frac{\partial \prod_{R}^{M, 3 P}}{\partial p}=0
$$

third party companies according to the manufacturer to determine the conversion price of $b$ to determine the recovery rate of waste products $\tau$, the optimal response to the solution:

$$
\operatorname{Max}_{\tau} \prod_{3 P}^{M, 3 P}=\left(\phi-\beta p^{2}\right)(b-A) \tau-L \tau^{2} .
$$

From $\tau$ to $\prod_{3 P}^{M, 3 P}$ has the pseudo concave, so the optimal reaction function is equivalent to the first order condition: 


$$
\frac{\partial \prod_{3 P}^{M, 3 P}}{\partial \tau}=0,
$$

the manufacturer's profit function is

$$
\prod_{M}^{M, 3 P}=\left(\phi-\beta p^{2}\right)\left(w-c_{m}+(\bar{\Delta}-b) \tau\right) .
$$

The (4.27) as the objective function, considering (4.24), (4.26) and other assumptions, recovery rate range set as the corresponding constraints is between 0 and 1 , building a model $M, 3 P$ nonlinear programming problems are as follows:

$$
\begin{aligned}
& \underset{\tau, p, b, w}{\operatorname{Max}} \prod_{M}^{M, 3 P}=\left(\phi-\beta p^{2}\right)\left(w-c_{m}+(\bar{\Delta}-b) \tau\right), \\
& (M, 3 P) \text { s.t. }\left\{\begin{array}{l}
3 \beta p^{2}-2 w \beta p-\phi=0, \\
\left(\phi-\beta p^{2}\right)(b-A)-2 L \tau=0 . \\
w+(\bar{\Delta}-A) \tau \geq c_{m} . \\
0 \leq \bar{\Delta} \leq \sqrt{\frac{\phi}{\beta}} \\
b \leq \bar{\Delta}, \\
p \geq w, \\
0 \leq \tau \leq 1 .
\end{array}\right.
\end{aligned}
$$

According to the objective function, it can be concluded that the objective function tends to the maximum value when $b^{* M, 3 P}=\frac{\bar{\Delta}+A}{2}$.

In the third party commissioned by the company recycling model, the total profit function

$$
\prod_{T}^{M, 3 P}=\prod_{M}^{M, 3 P}+\prod_{R}^{M, 3 P}+\prod_{3 P}^{M, 3 P}
$$


Similar to the 4.1 calculation, we have

$$
\begin{gathered}
p=\frac{\beta w+\sqrt{\beta^{2} w+3 \beta \phi}}{3 \beta}, \\
\tau=\frac{\left(\phi-\beta p^{2}\right)(b-A)}{2 L} .
\end{gathered}
$$

\section{Comparison and Selection Strategies of Three Recovery Modes}

\subsection{Relational comparison}

When the manufacturer does not directly recycling, commissioned by the retailer or third party companies recycling waste products will pay the price of $b$, from the last few sections of the analysis, we can see Table 1 results:

Table 1. Analytic solution of $b$

\begin{tabular}{|c|c|}
\hline Model $M, R$ & Model $M, 3 P$ \\
\hline$b^{* M, R}=\bar{\Delta}$ & $b^{* M, 3 P}=\frac{\bar{\Delta}+A}{2}$ \\
\hline
\end{tabular}

According to the results of the last section, the relationship between the optimal price and the optimal recovery rate of the three recovery models are listed, as shown in Table 2. 
Table 2. Optimal price and optimal recovery of the three recovery models

\begin{tabular}{|c|c|c|c|}
\hline Variable & Model $M, M$ & Model $M, R$ & Model $M, 3 P$ \\
\hline$p^{*}$ & $\frac{\beta w^{* M, M}+\sqrt{\beta^{2}\left(w^{* M, M}\right)^{2}+3 \beta \phi}}{3 \beta}$ & $\frac{\beta\left[w^{* M, R}-\left(b^{* M, R}-A\right) \tau^{* M, R}+\sqrt{\beta^{2}\left[w^{* M, R}-\left(b^{* M, R}-A\right) \tau^{* M, R}\right]^{2}+3 \beta \phi}\right.}{3 \beta}$ & $\frac{\beta w^{* M, 3 P}+\sqrt{\beta^{2}\left(w^{* M, 3 P}\right)^{2}+3 \beta \phi}}{3 \beta}$ \\
\hline$\tau^{*}$ & $\frac{\left(\phi-\beta p^{* M, M}=\right)(\bar{\Delta}-A)}{2 L}$ & $\frac{\left(\phi-\beta\left(p^{* M, R}\right)^{2}\right)\left(p^{* M, R}-w^{* M, R}+b^{* M, R}-A\right)}{2 L}$ & $\frac{\left(\phi-\beta\left(p^{* M, 3 P}\right)^{2}\right)\left(b^{* M, 3 P}-A\right)}{2 L}$ \\
\hline
\end{tabular}


We assume that no matter what kind of recycling mode, the production of the product quality is the same, when the direct recovery of manufacturers, retailers from the manufacturer to the wholesale price of product, unit cost is $w^{* M, M}$, per unit product of the retailers direct payment is $w^{* M, M}$. When the retailer's recovery, his unit cost is no longer a wholesale price, but wholesale price minus the retailers due to the recycling of waste products and obtained from the manufacturer of the compensation, per unit of product the actual cost is $W=B$, when retailers also must be considered for product recovery of fixed costs. When the retailer is actually spending $w^{* M, R}-\left(b^{* M, R}-A\right) \tau^{* M, R} \geq$ $w^{* M, M}$ per unit, the rational retailers consider whether to choose the recycling of waste products, so we only need to discuss the case of $w^{* M, M}>w^{* M, R}-\left(b^{* M, R}-A\right) \tau^{* M, R}$. In previous studies, this condition is satisfied under linear demand.

For the same reason, when a third party company for recycling, because manufacturers pay in addition to the manufacturing cost also includes the purchase of waste products of the conversion price, manufacturers in order to pass on this part of the spending will certainly wholesale price higher than their recovery is proposed, in order to compensate for this, when $w^{* M, 3 P}<w^{* M, M}$, not the manufacturers commissioned third-party companies to recycle waste products. At the same time, we need to discuss the:

$$
w^{* M, 3 P}>w^{* M, M} \text {. }
$$

That is,

$$
w^{* M, 3 P}>w^{* M, M}>w^{* M, R}-\left(b^{* M, R}-A\right) \tau^{* M, R} .
$$

\subsection{Propositional analysis}

On the basis of the comparative analysis, we can get the following proposition: 
Proposition 1. In the leading maker of bilateral monopoly market, three kinds of reverse logistics recycling mode $(M, M),(M, R)$, and $(M, 3 P)$, commissioned by the manufacturer retailer is responsible for recycling products and manufacturers directly recycled products than manufacturers commissioned third-party companies recovery when is more price advantage in the market, especially to the retailer recycling mode of price advantage is more significant. That is,

$$
p^{* M, 3 P}>p^{* M, M}>p^{* M, R} .
$$

As the recycling model $(M, R)$ has a strong price advantage, the choice of manufacturers to recycle is conducive to improving the product demand, there are:

$$
D p^{* M, 3 P}<D p^{* M, M}<D p^{* M, R} .
$$

Proposition 2. In three recycling models $(M, M),(M, R)$, and $(M, 3 P)$, the retailer recovery $(M, R)$ when the recovery is the most, and when the manufacturer commissioned third party recovery $(M, 3 P)$, the recovery rate is the lowest, that is,

$$
\tau^{* M, 3 P}<\tau^{* M, M}<\tau^{* M, R}
$$

Proposition 3. In the three modes of recycling, the manufacturer's profit in the retailer recovery model $(m, R)$ is the largest in manufacturer is responsible for recycling $(m, m)$ is smaller, in the manufacturer commissioned third-party companies responsible for recycling $(m, 3 P)$ minimum, that is,

$$
\prod_{M}^{* M, R}>\prod_{M}^{* M, M}>\prod_{M}^{* M, 3 P}
$$

Proposition 4. In the three recycling models, the retailer's profit is the largest in the direct recovery of manufacturers, when the retailer is recycling smaller, the third side of the company is responsible for the recovery of the minimum. That is,

$$
\prod_{R}^{* M, M}>\prod_{R}^{* M, R}>\prod_{R}^{* M, 3 P}
$$




\section{Simulation Mode}

In bilateral monopoly market structure, the leading maker, a plant to produce some electronic products implementation of the closed-loop supply chain of reverse logistics and the demand function presents nonlinear morphology: $D(p)=300000-1.2 p^{2}$ and manufacturers responsible for recycling of waste products, the manufacturing cost of the new product is $C_{m}=90$ (100 yuan), and the use of processing waste products re-manufacturing cost of the new product is: $C_{r}=74$ (100 yuan), the treatment by the manufacturer after the recovery of the waste products re utilization rate is: $R=0.1$ and cannot be used for remanufacturing of used products also has a residual value: $S=1$ (100 yuan). Simulation calculation is carried out, in order to facilitate the support of more data, our simulation respectively in the recovery of the scale parameter: $L=1000,2000,3000$, fixed cost of recycled products recycling respectively: $A=2.2,2.3,2.4$, by LINGO11 software to solve the three recycling mode of nonlinear programming global optimal solution, relevant results as shown in Table 3 shows: 
Table 3. Comparison of simulation results of 3 kinds of recycling channels

\begin{tabular}{|c|c|c|c|c|c|c|c|c|c|c|c|}
\hline $\begin{array}{c}\text { Market } \\
\text { scale }\end{array}$ & $\begin{array}{c}\text { Recovery } \\
\text { cost }\end{array}$ & $\begin{array}{c}\text { Recovery } \\
\text { model }\end{array}$ & $D p^{*}$ & $p^{*}$ & $w^{*}$ & $b^{*}$ & $\tau^{*}$ & $\prod_{M}^{*}$ & $\prod_{R}^{*}$ & $\prod_{T}^{*}$ & $\prod_{3 P}^{*}$ \\
\hline \multirow{9}{*}{$L=3000$} & \multirow{3}{*}{2.2} & $\mathrm{M}, \mathrm{M}$ & 5727 & 142.22 & 125.45 & & 0.286 & 203241 & 96077 & 299318 & \multirow[b]{3}{*}{61.42} \\
\hline & & $\mathrm{M}, \mathrm{R}$ & 5742 & 142.18 & 126.39 & 3.24 & 1.00 & 204715 & 93638 & 298353 & \\
\hline & & $\mathrm{M}, 3 \mathrm{P}$ & 5723 & 142.23 & 125.47 & 2.35 & 0.143 & 203118 & 95964 & 299144 & \\
\hline & \multirow{3}{*}{2.3} & $\mathrm{M}, \mathrm{M}$ & 5723 & 142.23 & 125.47 & & 0.191 & 203104 & 95952 & 299056 & \multirow[b]{3}{*}{27.28} \\
\hline & & $\mathrm{M}, \mathrm{R}$ & 5735 & 142.20 & 126.44 & 3.35 & 1.00 & 204141 & 93376 & 297516 & \\
\hline & & $\mathrm{M}, 3 \mathrm{P}$ & 5722 & 142.24 & 125.48 & 2.40 & 0.095 & 203050 & 95902 & 298979 & \\
\hline & \multirow{3}{*}{2.4} & $\mathrm{M}, \mathrm{M}$ & 5721 & 142.24 & 125.48 & & 0.095 & 203022 & 95877 & 298899 & \multirow[b]{3}{*}{6.82} \\
\hline & & $\mathrm{M}, \mathrm{R}$ & 5728 & 142.22 & 126.49 & 3.45 & 1.00 & 203568 & 93114 & 296681 & \\
\hline & & $\mathrm{M}, 3 \mathrm{P}$ & 5721 & 142.24 & 125.48 & 2.45 & 0.048 & 203009 & 95864 & 298880 & \\
\hline \multirow{3}{*}{1000} & \multirow{9}{*}{$A=2.3$} & $\mathrm{M}, \mathrm{M}$ & 5729 & 142.22 & 125.43 & & 0.573 & 203323 & 96152 & 299475 & \\
\hline & & $\mathrm{M}, \mathrm{R}$ & 5735 & 142.20 & 125.74 & 2.65 & 1.00 & 204141 & 95376 & 299516 & \\
\hline & & $\mathrm{M}, 3 \mathrm{P}$ & 57825 & 142.23 & 125.46 & 2.40 & 0.286 & 203159 & 96002 & 299243 & 81.93 \\
\hline \multirow{3}{*}{2000} & & $\mathrm{M}, \mathrm{M}$ & 5725 & 142.23 & 16.77 & & 0.286 & 203159 & 96002 & 299161 & \multirow[b]{3}{*}{40.93} \\
\hline & & $\mathrm{M}, \mathrm{R}$ & 5735 & 142.20 & 126.09 & 3.00 & 1.00 & 204141 & 94376 & 298516 & \\
\hline & & $\mathrm{M}, 3 \mathrm{P}$ & 5722 & 142.24 & 125.44 & 2.40 & 0.143 & 203077 & 95927 & 299045 & \\
\hline \multirow{3}{*}{3000} & & $\mathrm{M}, \mathrm{M}$ & 5723 & 142.23 & 125.47 & & 0.191 & 203104 & 95952 & 299056 & \multirow[b]{3}{*}{27.28} \\
\hline & & $\mathrm{M}, \mathrm{R}$ & 5735 & 142.20 & 126.44 & 3.35 & 1.00 & 204141 & 93376 & 297516 & \\
\hline & & $\mathrm{M}, 3 \mathrm{P}$ & 5722 & 142.24 & 125.48 & 2.40 & 0.095 & 203050 & 95902 & 298979 & \\
\hline
\end{tabular}


From Table 3 and model results come to the same conclusion, and in the recovery of fixed costs and scale parameters are consistent, commissioned by the manufacturer retailer recycling $(M, R)$, consumers can gain the maximum profit. Because of the lowest selling price, for manufacturers, the $U$ recovery mode optimal efficiency. The impact of the social environment, the highest recycling efficiency of retailers, can bring greater positive and effect to the environment. This recycling model can take into account the comprehensive benefits of economy and environment, not only conducive to the sustainable development of enterprises, but also contribute to the sustainable development of the society as a whole.

\section{Model Result Analysis}

Due to the complexity of the calculation of the nonlinear programming problem, can not through mathematical derivation completely describe the three recycling model of closed-loop supply chain channel overall profit, we refer to the derived proposition, and the simulation results comparison, some conclusions are drawn as follows:

Conclusion 1. In the case of all parameters consistent

$$
\tau^{* M, R}>\tau^{* M, M}>\tau^{* M, 3 P}
$$

The recovery rate of the size reflects the reverse logistics recycling mode of success or not, in the leading maker of bilateral monopoly market, the retailer is responsible for recycling $(M, R)$ recovery rate is highest, manufacturers direct recovery $(M, M)$ times the recovery efficiency is the lowest is the third party company responsible for recycling $(M, 3 P)$ mode. Because the third party company for recycling, he won the marginal profit of $\left(b^{* M, 3 P}-A\right)$, and manufacturers to recycle

the marginal profit $(\bar{\Delta}-A)$, because $\left(b^{* M, 3 P}-A\right) \leq(\bar{\Delta}-A)$, so thirdparty companies on the recovery of investment to gain than the direct 
recovery of manufacturers is less profitable. In addition, manufacturers can also be directly recovered through the retailer's pricing strategy to promote the recycling of waste products, resulting in the recovery of the two level effect. This phenomenon is consistent with the linear demand and nonlinear demand of the product.

Conclusion 2. In the case of all parameters consistent,

$$
p^{* M, R}<p^{* M, M}<p^{* M, 3 P}, D p^{* M, R}>D p^{* M, M}>D p^{* M, 3 P} .
$$

By retailer recycling $(M, R)$ when the sales price is the lowest, the second is the manufacturer directly recycling $(M, M)$, the highest is when the third party companies to recover $(M, 3 P)$. Market demand and sales price is inversely proportional to the relationship, so the market demand for the relationship between the performance of: $D p^{* M, R}>D p^{* M, M}>D p^{* M, 3 P}$. In monopoly market leading manufacturer and the third party company profit only in the third party company for recycling is likely to emerge, but third-party companies cannot directly enjoy recovery reverse logistics or remanufacturing produce excess profits, he obtained the profit is the manufacturer and the retailer enjoyed remanufacturing profit margins. For manufacturers, he can get more profit from the lower retail price, because the manufacturer will be able to produce a lower wholesale price, which will benefit from increasing market demand. When recovery for retailers, retail prices in all recycling channels lowest retailers through his pricing strategy to influence market demand, and direct profit from, so manufacturers authorized dealer for recycling, the demand for a product. This result is consistent with the conclusions drawn from the study of the recovery model based on linear demand.

Conclusion 3. In the case of all parameters consistent,

$$
\prod_{M}^{* M, R}>\prod_{M}^{* M, M}>\prod_{M}^{* M, 3 P} ; \prod_{R}^{* M, M}>\prod_{R}^{* M, R}>\prod_{R}^{* M, 3 P}
$$

When the recovery rate and the lowest price, the market demand of the products is maximum, as the main body of the closed-loop supply chain member manufacturers to maximize profits. Through the analysis 
of the above comparative simulation, in the structure of the market leading manufacturers, manufacturers commissioned recovery mode when the best retailers. But the retailer is not in $(M, R)$ in the most profitable. The profits of the whole channel is not necessarily in direct recovery when the largest manufacturer.

Conclusion 4. In the case of all the parameters, with the increase of the degree of difficulty of the recovery $L$ and the recovery of the fixed cost of $A$ increased, resulting in $\tau^{*}$ decrease, $p^{*}$ increase, $D p^{*}$ decrease, $\prod_{M}^{*}, \prod_{R}^{*}, \prod_{3 P}^{*}$, and $\prod_{T}^{*}$ are reduced.

Conclusion 5. In all cases with the same parameters, the leading manufacturer monopoly market, entrusted by the manufacturer retailer $(M, R)$ recovery is the optimal decision makers.

Because the high recovery rate means that the environmental contribution is large, low sales prices means that the majority of consumer groups benefit. These variables are in the retailer recycling mode $(M, R)$ in the best performance. So $M, R$ model is the optimal decision. Even though our simulation results show that the overall profit of the channel in this model does not have the highest, but the closed-loop supply chain in addition to the pursuit of economic profits, but also take into account the impact on the environment, from the implementation of the closed-loop supply chain need to select, $(M, R)$ is optimal decision.

\section{Coordination Optimization Analysis of $(M, R)$ Model}

From the above research, we draw the conclusion: the entrusted by the manufacturer retailer $(M, R)$ recovery mode is a leading manufacturer recycling mode under the monopoly market of the most effective implementation of the closed-loop supply chain. 
In $(M, R)$ recycling model: because there are $\prod_{M}^{* M, R}>\prod_{M}^{* M, M}>$ $\prod_{M}^{* M, 3 P}$, so manufacturers are willing to choose to entrust the retailer for recycling, but there are $\prod_{R}^{* M, M}>\prod_{R}^{* M, R}>\prod_{R}^{* M, 3 P}$, and in some cases $\prod_{R}^{* M, R}$ close to $\prod_{R}^{* M, M}$.

In order to successfully implement the closed-loop supply chain, as a market leader in the manufacturer in order to make a profit, he should be paid by the additional recycling subsidies to encourage retailers to recycle. The corresponding coordination mechanism is as follows:

Manufacturers and retailers have negotiated a distribution coefficient $\alpha$, of which $0<\alpha<100$. When

$$
F=\prod_{M}^{* M, R}-\prod_{M}^{* M, M}>\prod_{R}^{* M, M}-\prod_{R}^{* M, M}
$$

In this model, $M$ only take the profit $\prod_{M}^{* M, R}-F \alpha \%$, when $R$ take $\prod_{R}^{* M, R}+F \alpha \%$, because the $F$ is relatively large, so $\prod_{R}^{* M, R}+F \alpha \%>$ $\prod_{R}^{* M, M}$.

When the manufacturer is assigned to the retailer from the profit $F \alpha \%$ distribution, the retailer will consider the commitment to recycling business. At the same time as a government agency, in order to environmental guidance should also be through government subsidies and other means to encourage retailers to open up recycling channels, so that $(M, R)$ model to achieve coordinated and optimized objectives. 


\section{References}

[1] R. C. Savaskan, S. Bhattacharya and L. Van Wassenhove, Closed-loop supply chain models with product remanufacturing, Management Science 50(2) (2004), 239-253.

[2] R. C. Savaskan and L. Van Wassenhove, Reverse channel design: The case of competing retailers, Management Science 52(1) (2006), 1-14.

[3] G. U. Qiaolun, G. A. O. Tiegang and S. H. I. Lianshuan, Price decision analysis for reverse supply chain based on game theory, Systems Engineering Theory \& Practice (3) (2005), 20-25 (in Chinese).

[4] Guohua Sun, Qiushuang Chen, Haitao Xu et al., Coordination \& decision-making between manufacturers and retailers in integrated remanufacturing/manufacturing system, Computer Integrated Manufacturing System 12(1) (2005), 127-132 (in Chinese).

[5] Huang Zuqing and Qingli Da, Study on efficiency of serial supply chains with remanufacture, Journal of Management Sciences in China 9(4) (2006), 51-57 (in Chinese).

[6] Xiaohua Han and Shengjia Xue, Coordination mechanism analysis for closed-loop supply chain under asymmetric information, Computer Integrated Manufacturing System 14(4) (2008), 731-736 (in Chinese).

[7] Xiaohua Han and Shengjia Xue, Reverse channel decision for competing closed-loop supply chain with dominant retailer, Computer Integrated Manufacturing System 15(11) (2009), 2247-2253 (in Chinese).

[8] Shengjia Xue, Some methods to constitute pseudoconvex (pseudoconcave) functions, Journal of Guangxi University (Natural Science), (1) (1980) 97-101 (in Chinese).

[9] Shengjia Xue, Notes on stability analysis in mathematical programming, Journal of Jinan University (Natural Science) 19(3) (1998), 15-20 (in Chinese). 